\title{
Yunnan Hani dance posture analysis In Yuanjiang -County Sheep Street Xiang Fan dance as an example
}

\author{
Yuting $E^{1, a}$ \\ ${ }^{1}$ Yuxi Normal University, Yuxi, Yunnan, 653100 \\ ${ }^{a}$ email
}

\begin{abstract}
Keywords:Yuanjiang County Sheep Street; Xiang Hani fan dance; dance posture;causes
\end{abstract}
\begin{abstract}
The generation, formation and shaping of a national dance and its natural environment and social and historical development are not separated from the natural environment and social history. Yunnan Yuanjiang County Sheep Street Xiang Hani nationality Brown fan dance and knees bent, leaning forward, containing in body features formed by Hani social development process in the history of migration, the farming life, traditional moral values and customs.
\end{abstract}

\section{Introduction}

Dance posture is the basic form of dance, it mainly refers to dance when the trunk of the human body with what kind of posture, such as forward or backward, straight line or curve. Ethnic dance posture has the typical characteristics of national culture, such as the representative body of the dais dancing squat state of three curved shape, it exhibits lived in accordance with the water, like peacock, longing for peace, to believe in Hinayana Buddhism based national characteristics; and Mongolian Dance representative body features with a tall and straight, after supine and horizontal momentum, it represented the life on the vast grassland, riding the hegemony of Eurasian nomadic characteristics.

The generation, formation and setting of a national dance body are not separated from the natural environment and the social and historical development of its existence. According to the survey, the physical characteristics of Yunnan Yuanjiang Yang Jie Xiang Fan dance mainly to bend the knee, forward, chest.

People not only have natural attributes, but also social attributes. Human body also has natural attributes and social groups. Different people in different ethnic groups, social and cultural systems have their own characteristics. Body in the inheritance of cultural and social attributes, but also the continuation of its natural attributes. Body first determines the constitution. Constitution is the inherent characteristic of the human body on the basis of hereditary acquired function and the relative stability of morphology. The Constitution can be classified according to the morphology, function or metabolic characteristics of human." [1] according to the analysis of physical anthropology, "modern Hani ethnic group is China's southern hairstyle and euryprosopic, nose, short physical. Because of the history of the ethnic minorities in the migration, environment, water and soil, the living habits of the change, ethnic marriages, so that the nation's physical characteristics are disappearing, and gradually close to other ethnic groups. The influence of ethnic origin and geographical factors on the physical characteristics of each nation is weakening." [2] therefore, the Yang Jie Xiang led body form of research, especially in the external environment factors and the social and historical development process of Yang Jie Xiang Hani nationality Brown fan dance posture effect, while ignoring Yang Jie Xiang Hani body constitution itself structure analysis.

Hani with today in Yunnan Province more than a dozen Yi branch of the people, the mainstream are homologous in the ancient Diqiang clans. The ancient Diqiang nomadic tribes, who in the northwest region "China river source" Qinghai, Gansu, Tibetan plateau. Ad in the first three centuries, including Hani ancestors, the ancient Qiang people is in the Hexi Corridor, along the coast of Lake Qinghai "NUMA may" grassland. Diqiang culture of that period although not entirely representative of the culture of the Hani ancestors, but the analogy, from can be derived Yang Jie Xiang Hani nationality Brown fan 
dance body formation reasons. 1973 unearthed in Datong County, Qinghai Province Sunjiazhai dance grainpainted pottery basin (51 years ago to 4700 years) the family problem, although controversial, but many scholars think manufacturing in Datong County, Qinghai Province dance painted pottery basin of ancestors and the ancient Qiang related. In the basin, there are 3 groups of people, 5 people in each group. Dance people around the basin wall to form a circle, hand in hand, the arrangement is neat, the action is consistent. From the dance shape, dance knee flexion micro foundation, rise. From the dance force, head look right and left body micro back, seems to be in the counterclockwise direction of dance. The ancient Diqiang nomadic group was in the vicinity of Qinghai. Man has his head up to look far and wide. Cattle and sheep grazing must to the distance overlooks, life, long-term grazing, ancient Diqiang ethnic groups of human conduct rise after supine posture. Qinghai is located in the northeastern part of the Tibetan Plateau, towering mountains, diverse terrain, rivers, lakes. In the process of walking, in total knee flexion and extension. If the knee stretched too straight, easily tired and easily injured. The knee walking in the process of long-term in a state of micro bend. The life needs to continue to pull mountain wading, walking behavior for a long time the ancient Diqiang ethnic groups to form a micro knee bent posture. Today Yang Jie Xiang traditional Hani nationality Brown fan dance posture showing the knees, leaning forward, including chest, and unearthed from the Sunjiazhai Datong County, Qinghai Province dance grainpainted pottery dance knee microbend flexion, conduct the rise, after the Yang of the body have a connection, but with a difference. The relationship was reflected in the knee flexion state, although the degree of flexion is not consistent with the. The difference is reflected in the upper body, the former forward with the chest, and then the rise of the latter. The difference is not to say that they have no internal relations, but the historical development of the natural and social factors leading to the formation of the Hani dance body changes.

\section{The migration caused by changes in body}

From the NUMA may (now the Jinsha River, the source of the Yangtze River in the northern Tibetan Plateau a macrophyte abundance prairie) to Lassa fan Jenna Jin Yuanjiang about 1600 kilometers of straight-line distance. Although the Hani ancestors has in Ecuador for Niang (now Dali Erhai Lake area), Lur Hamid Char (now Kunming Dianchi Lake area), were to stay, but after dozens of generations long trek, the Hani ancestors gradually adapt to the walk of life, and form suitable for long-distance walking posture. In the high peaks and valleys of the Hengduan Mountains shuttle, from north to South gallop and pass the rivers of ice from the water, long-distance Bashansheshui, so that a more developed the legs of the Hani ancestors, strong, elastic, the degree of knee flexion is more obvious. Because migration is mostly due to war or living environment caused by the deterioration, the Hani ancestors in the background every time away from home re embark on the journey has to take the necessities of life, with family and livestock in a hurry. Back old, weak, sick and disabled, cradling baby baby, shoulders holding material, holding the hands of cattle, seeing the road, at the foot of the stable every step of the way. Shoulders bowed down with the weight of the Hani ancestors originally upright Shenqu, embrace the flat chest containing cupped inward, and in order to stabilize the foot. After the original supine posture must forward was visible at the foot of the rugged road, won't cause the items back down. In addition, due to the lost their homes and loved ones with a heavy heart disease and defeat the Hani ancestors by the original upright, supine posture change for forward and downward dragging a heavy pace forward posture.

\section{The cultivation of the body shape}

In Yuanjiang, Hani ancestors had settled on a stable life. Yuanjiang rich products and humid, hot weather, and live in Ba Zi Dai mature rice for methods and techniques, Hani ancestors with the nomadic rich experience accumulated in the process of quickly into the settled agricultural production and social. Due to population growth, part of the Hani ancestors left Yuanjiang Bazi to "the white" (Xinping, Chuxiong, Dali direction), to "Lasha Roba" (today's Red River, Yuanyang direction), to the sun direction 
(now Pu'er, Jinghong direction) to move out. To this Sheep Street, the village of Hani ancestors according to Sheep Street, mountain high water and high geographic conditions, along the towering clouds of Ailao Mountain from the foot to the top of the hill, the repair of the terraces of the sky line. Hani ancestors daily living in the hillside to take pole on terrace miles even tens of miles. Layers of terraced fields, Geng Hui Gou turn, row upon row of walking in such fields, labor, attention should be paid to the knees remain some flexion, in order to protect the knee is not due to stretch too straight and the bone marrow collision damage. Therefore, terraced fields, especially the whereabouts of knee action is formed the habit of daily life is fixed to the body of Hani ancestors. The knee is Custom makes all things easy. appear in the dance posture. The pole for people living in mountainous and semi mountainous area is an important method to transport materials. Hani ancestors from using it as a tool of labor, whether to carry other tools to work in the fields, or the food home, or with children on the terrace, it is less Hani men can not transport.

Shoulder long-term under heavy pressure, not only to suppress the person's body to grow taller, but also created a chest containing body. And a man with shoulder, back and the basket is Hani women ancestors indispensable means of transport. Hani women head back firewood basket carried on the back, arms holding or hugging baby hand carrying home cooking vegetables and can walk the child is common. Hani women first civilian their broad mind and hard-working hands raised countless Hani people, creating a magnificent Hani field at the same time, they also created a small, embedded body. Back back and the basket is the main force with the forehead top back in the back of the material. At this time the body must be from the beginning of the body forward lean forward to the back of the items will not fall. When the back of the goods is too heavy, and they must more bending forward to the power balance, do not cause a person to be pulled down the back of the goods. Heavy pressure not only oppressed the knees of Hani ancestors, also oppressed their upper body, so that their upper body forward, rickets. That in the burdens, the forward, chest posture also exists in their life. Even the most relaxed palm fan dance entertainment moment is also lingering. Yang Jie Xiang Hani lived in semi hillside area, as long as go out, whether labor or neighbor on the way home, uphill. In the uphill normal human body is forward, one of the important reasons for this is the sheep Jie Xiang Fan Dance 。

\section{The traditional moral concepts and customs and habits}

An important reason for the formation of Hani ancestors of traditional moral values and customs is also a Sheep Street Xiang Zong fan dance posture, leading to Yang Jie Xiang Fan Dance posture solidified. Hani family nursing, filial piety old traditional morality, and derived therefrom, the father and son linked name of, the Hani ancestors attention blood relationship, the external row of his psychological structure has an important influence. Hani of different materials and traditional very sensitive, will be regarded as a prelude to disaster or ominous signs and force rejection. In the fan dance is included, limbs, bending forward, back, chest wrapped body force on the arms control arc. At the same time, with the elders too much respect for formation of servile form, and to the exclusion of foreign invaders, psychological resistance and Sheep Street, Xiang led in, bent body formed. In addition, commensurate with Hani ancestors, thrifty, plain and concise values and patriarchal ethics and a series of custom is also a Sheep Street Xiang Zong fan dance posture formed one of the reasons. Such as Hani peacetime life simple, breakfast eat early, eat dinner late, went out at noon labor can not go home to dine, general to cold food to the labor side or the side of the field shacks squatting to eat. Since entering the patriarchal clan society, the Hani women's status is relatively low. Even at home, often in the dining room, pond edge outside the aisle to eat. If the father-in-law in the church, do daughter-in-law can only squat meal in law. And as the Hani ancestors are generally cooked in the fire support tripod pot. Because the fire on the ground, so the Hani ancestors will bend over, knees to cook. More than forward bends, kneeling or squatting movements are formed Yang Jie Xiang Hani nationality Brown fan dance forward, with chest and knees posture natural portrayal of life.

\section{Conclusion}


Relating to the above Yuanjiang County Sheep Street Xiang Zong fan dance and knees bent, leaning forward, containing in the body features of origin from the analysis can be seen: in Yuanjiang County Sheep Street, Xiang led the historical evolution process, the main reason for the formation of Yuanjiang County Sheep Street Xiang Zong fan dance posture, Hani social historical development in the long-term migration, farming life and traditional moral values and customs. From the reflection of the epic Hani and overall social and historical changes of the Hani APEI slope ", and today in the terraced fields of farming as the main, still in continuous southward migration, which still influence the Hani social development of traditional moral concept and custom of Hani social reality, and many ha a Shawnee dance with knees bent, leaning forward, with in the body features, can be inferred: Hani's history of migration, farming life and traditional moral values and customs is the main factors affecting Hani dancing posture development.

\section{References:}

[1] Shanghai: [S]. Hai Hai Shanghai dictionary press, 2000.

[2] Li Ming, Wang Xiaoqing, Sun Jun et al. The physical characteristics of the 14 ethnic groups in Yunnan [J]. Shanghai: Journal of anatomy, 2005 